\title{
Negative contrast after prolonged discrimination maintenance
}

G. S. REYNOLDS AND A. J. LIMPO

UNIVERSITY OF CALIFORNIA, SAN DIEGO

Negative contrast occurs during the abolishment of a discrimination between two stimuli even after prolonged maintenance of the discrimination virtually eliminates positive contrast.

Terrace (1966) has reported that prolonged maintenance of a discrimination between $S+$ and $S-$ results in the disappearance of positive behavioral contrast. After increasing during the formation of the discrimination (contrast), the rate of responding during $S+$ decreases to, or at least toward, its prediscrimination level. Since Terrace has interpreted contrast as owing to an emotional frustration generated by extinction during S-, he has interpreted the disappearance of positive contrast over time as owing to the habituation of the emotion. Both the appearance and disappearance of behavioral contrast are thus explained by appeal to an intervening, emotional effect of changes in the frequency of reinforcement.

The present paper casts doubt on this explanation. Negative contrast (Reynolds, 1961a, 1961b), which is presumably due to a disappearance of the emotion generated by nonreinforcement, is studied after prolonged maintenance of a discrimination has already, according to the theory, allowed the disappearance of the emotion.

Subjects

Six pigeons were maintained at $80 \%$ of free-feeding weight.

Apparatus

The experimental space, with a single key transilluminable by red or green light, was connected to automatic programing and recording equipment.

Procedure

Each daily session consisted of alternating red and green illumination of the key. The duration of each stimulus was either 15, 30, or 45 sec. Each duration occurred a total of eight times for each of the stimuli in an irregular order during the 24 min session.

At first, pecking was reinforced in the presence of each color on a variable-interval schedule with an average inter-reinforcement interval of $30 \mathrm{sec}$. When stable and equal rates of responding prevailed during each stimulus, pecking was extinguished for 92 sessions during the red key for three birds and during the green key for three birds. Finally, in order to observe negative contrast, responding was reinforced during both stimuli, for four sessions.

Results

Figure 1 shows the rate of responding in the presence of $S_{+}$for each of the six birds during the last four sessions ( 89 to 92 ) of maintenance of the discrimination (to the left of the vertical dashed line in each panel) and the rate of responding in the presence of the same stimulus during the following four sessions of reinforcement in the presence of both stimuli (to right of this dashed line). The notation on the abscissa indicates the schedule of reinforcement associated with each color during each block of four sessions. Negative contrast is seen in the data of each bird as a decrease in the rate of responding during the second group of four sessions.

Figure 2 summarizes these data by showing the average rate of responding as a function of daily sessions under the two conditions of the experiment, indicated on the abscissa. Even after prolonged maintenance of a discrimination, the rate of responding decreases in the presence of St when responding is
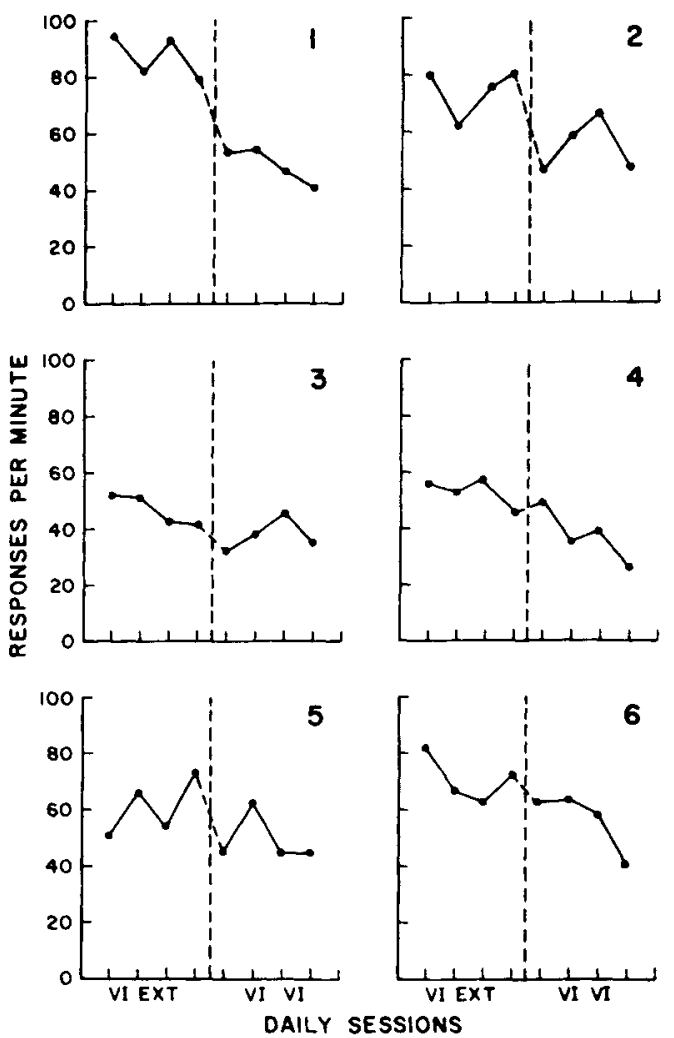

Fig. 1. The rate of responding in the presence of $S+$ during the last four sessions of maintenance of a discrimination (left of dashed line) and the first four sessions of its abolishment (right of dashed line) for six pigeons. 


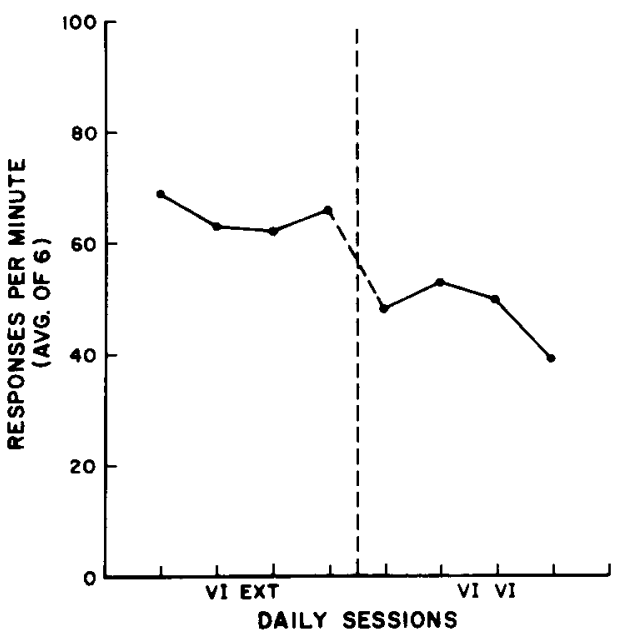

Fig. 2. Averages of the rates of responding shown in Fig. 1.

once again reinforced in the presence of the other stimulus.

\section{Discussion}

From previous (Reynolds, 1961a, 1961b; Terrace, 1966), as well as the present data, it appears that the sequence of events during the formation and abolishment of a discrimination between two stimuli is as follows. At first, when responding is reinforced in the presence of each of two stimuli, the rates of responding in their presence are approximately equal. When responding is extinguished in the presence of one stimulus (S-), the rate of responding declines in extinction during $\mathrm{S}-$, and the rate of responding during St increases, even though there has been no change in the conditions of reinforcement prevailing in the presence of S+. If the discrimination is now maintained for a period of some 60 to 90 sessions, the rate of responding in the presence of St gradually decreases toward its prediscrimination level; the magnitude of positive behavioral contrast decreases during prolonged maintenance of the discrimination. Finally, if responding is once again reinforced in the presence of each of the two stimuli, the rate of responding of course increases during the stimulus previously associated with extinction and the rate in the presence of $\mathrm{S}_{+}$ decreases still more. Whether or not this negative behavioral contrast will itself attenuate over a large number of subsequent sessions is currently not known, but it would seem likely. Hence, the process of discrimination formation appears to entail two relatively large, but in the long run transient, variations in the rate of responding prevailing in the presence of a stimulus whose conditions of reinforcement remain unchanged. One is positive contrast; the other is negative contrast.

Attempts to explain contrast by appeal to an emotion aroused by nonreinforcement or nonreinforced responding, while they can explain a negative contrast if it follows a large positive contrast soon after the formation of a discrimination, can hardly account for negative contrast after prolonged discrimination when positive contrast has all but disappeared. In order to do so, one would have to say that the emotion is aroused by nonreinforcement and results in an increase in the rate of responding during $\mathrm{S}+$; that it is thereafter habituated and allows a decrease in the magnitude of positive contrast with time; and finally that the affect is somehow reversed in sign by the reoccurrence of reinforcement and produces negative contrast. Whatever its original appeal, the theory becomes inevitably stretched and less believable by such an extension. It invites endless explanation of phenomena by appeal to concomitantly aroused, usually unobservable events, including emotions-by appeal to affect instead of effect. Positive and negative contrast are better understood as orderly phases in the dynamics of responding in the presence of a stimulus under changes in the conditions of responding and reinforcement in the presence of a different stimulus.

References

REYNOLDS, G. S. Behavioral contrast, J. exp. Anal. Behav., 1961a, 4, $57-71$.

REYNOLDS, G. S. An analysis of interactions in a multiple schedule, J. exp. Anal. Behav., 1961b, 4, 107-117.

TERRACE, H. S. Behavioral contrast and the peak shift: effects of extended discrimination training. J. exp. Anal. Behav., 1966, 9, 613-617.

Note

1. This research was supported by NSF Grant GB-2541 to the Univer sity of Chicago and by NSF Grants GB-5064 and GB-6821 to the University of California, San Diego. Reprints may be obtained from the first author, Department of Psychology, UCSD, P. O. Box 109, La Jolla, California 92037. 